\title{
Modelling Facility Location Problems in Emerging Markets: The Case of The Public Healthcare Sector in Morocco
}

\author{
Asmae El Mokrini \\ Rabat Business School, BEAR-Lab, \\ International University of Rabat, Morocco \\ Email: asmae.elmokrini@uir.ac.ma \\ Youssef Boulaksil \\ College of Business and Economics, \\ UAE University, Al Ain, United Arab Emirates \\ Email: y.boulaksil@uaeu.ac.ae ; youssef.boulaksil@gmail.com (Corresponding Author)
}

\author{
Abdelaziz Berrado \\ Research team AMIPS, EMI, \\ Mohammed V University, Rabat, Morocco \\ Email: berrado@emi.ac.ma
}

\begin{abstract}
Locating facilities in an emerging market involves specific characteristics that are different from developed countries. The differences between these two settings suggest that facility location modelling can present substantially different challenges when applied to an emerging market as opposed to a developed country. In this study, we develop a variant of the set covering model that explicitly takes specific characteristics into consideration. The model has been constructed and validated based on a real-life case study concerning the Moroccan Ministry of Health which is currently considering the redesign of its supply chain of pharmaceutical products. Amongst the findings, this paper shows that road infrastructure and demand dispersion affect the strategic decision of facility locations.
\end{abstract}

Keywords: facility location, emerging economies, healthcare, supply chain (re-)design

\section{INTRODUCTION}

The aim of facility location models is to develop an appropriate network for improved reactivity and capacity of the supply chain. Locating facilities inadequately can lead to increased costs or bad customer service in industry. Customer service can be greatly affected if the location of facilities is not set appropriately, even if their number is correctly determined (Daskin and Dean, 2005). Modelling facility location problems is governed by the contextual setting of the area under study. Literature shows that the applications of location-allocation models are frequently found in the context of developed countries [Amin and Zhang (2013), Batta et al., (2014)]. However, applications to emerging markets received little attention. Taking into consideration the differences between these two settings, location problems can be substantially different from facility location models in developed countries. The difference is mainly a result of environments where infrastructure configuration, society dynamics, technological capabilities, maturity levels, and strategic purposes show significant variances. We find that only few studies have taken specific characteristics of emerging markets into consideration. The aim of this study is to present a model that takes into consideration specific characteristics that can present an issue in an emerging market. Specifically, we would like to know how road infrastructure type and demand dispersion affects facility location decisions.

Optimization problems in healthcare have received significant attention in the past few decades (Rais and Viana, 2011). In particular, our model has been applied to a real-life case study that suggests a redesign for the pharmaceutical supply chain of the Moroccan Ministry of Health. The implications of poor location decisions in healthcare extend well beyond cost criteria. As an emerging market, the Moroccan health sector specificities and geographical setting can impact how warehouses are located. Demand levels and their distribution can also influence the decision. Amongst the findings of this study, we show how infrastructure type and demand dispersion affect the strategic decision of facility location. The remainder of this paper is organized as follows. In the next section, a review of relevant literature about facility location models is presented, highlighting the differences between emerging and developed countries. In section 3, the proposed model is presented. In Section 4 , the case study is presented as well as its results and a sensitivity analysis.

\section{LITERATURE REVIEW}

The literature on facility location models is rich; many models have been developed to assist the decision maker in deciding about the optimal location of facilities. Several surveys and literature reviews present facility location 
problems (Melo et al., 2009, Eiselt and Marianov, 2011, Arabani and Farahani, 2012, Drezner and Hamacher, 2001, Snyder, 2006), Şahin, and Süral, 2007). Facility location models can be generally classified into these four categories: analytical models, continuous models, network models, and discrete models (ReVelle et al. 2008). Analytical modelsdescribe systems as mathematical models by making simplified assumptions regarding the demand distribution or the cost structure of the network while giving exact solutions (Mousavi et al, 2013). In continuous models, facilities can be located at any point on a continuous space while demand is typically concentrated in points (Carlsson and Jia, 2014). The selection of sites for new facilities in discrete models is limited to a finite set of available candidate locations (Nickel and Gama, 2015; Kochetov, 2011; Schütz et al., 2008). Network problems, on the other hand, present models where facilities and customers are positioned on a network and demand is typically associated to nodes (Daskin, 2011). In approaching facility location problems in the specific context of healthcare (e.g. Mousazadeh et al., 2018), the literature shows that the three most used models are the location set covering model, maximal covering model, and p-median model (Daskin and Dean, 2005). The p-median model aims at minimizing the travelling distance where the farthest consumers can be put at disadvantage, which is usually encountered in rural health facilities for developing nations (Rahman, 1991). The coverage concept was first introduced by Toregas et al. (1971). Using this approach, a customer can be served if a facility is located within a given threshold distance or travel time from it (Francis et al., 1992). This threshold is called the coverage distance or coverage radius (Fallah et al., 2009). Schilling et al. (1993) classify models which use the concept of covering in two categories: 1) Set Covering Problem (SCP) where coverage is required and tries to minimize location cost while satisfying a specified level of coverage. 2) Maximal Covering Location Problem (MCLP) where coverage is optimized and maximizes the amount of demand covered within the acceptable service distance by locating a given fixed number of new facilities.

Facility locations models present differences from one sector to another. For example, the private sector's objective is generally the minimization of costs or the sum of the distances to the customers, while the public sector attempts to apply fairness and equity to all citizens while minimizing costs. Then, one of the aims would be the minimization of the distance to the furthest customer. This means that distances to other customers may increase so that all customers benefit from a minimum covering distance (Eiselt and Marianov, 2011). The problem of facility location typically arises when dealing with network decentralization decisions because such decisions require determining the optimal number and locations of facilities (ex. Mitropoulos et al., 2016). Several governments have attempted to decentralize public facilities in order to increase citizens' service performance levels (Common et al, 2016). Decentralization has been a prominent organizational reform in the government activities of emerging markets in recent years and activities in many sectors, including health, have been decentralized (Bossert et al., 2003; Thunhurst, 2012). The effect of a given pattern of decentralization depends on the context in which it occurs, including aspects of geographical configuration, political accountability, technical skill, and cultural norms. As a result, the same reform can produce different results in different countries (Roberts, 2011). Arney et al. (2014) state that the pharmaceutical distribution system in particular in most emerging markets has a different market structure compared to developed countries. The main differences include a lack of distribution networks with national reach, excessive fragmentation and many small players, many intermediaries between the manufacturer and the patient, and poor IT and communication flow systems resulting in poor coordination across actors in the distribution channel. Facility location modelling can then be affected by the differences between emerging markets and developed countries regarding the nature of facilities and their sector of activity.

The literature shows several implementations of facility location models pertaining to the healthcare sector in developed countries (Beheshtifar and Alimoahmmadi, 2015; Laporte et al., 2015; Haase and Muller, 2015; Han, 2014; Khodaparasti et al., 2016). Drezner and Hamacher (2001) state that the implementation of location-allocation problems in emerging markets is different from that of developed countries, mainly because of data limitation. In fact, applications of facility location to emerging markets show that several characteristics of these countries are taken into consideration at the modelling stage such as travel time, level of education, neighbourhood accessibility, etc. While studies pertaining to developed countries usually include coverage distance as the unique decision factor (Mitropoulos et al., 2016). For example, Buor (2003) takes into consideration distance in accessing health facilities in a rural district of Ghana in relation to other factors such as travel time, cost and level of education. Banerji and Fisher (1974) used the pmedian and set-covering model to hierarchically locate facilities for integrated planning in micro-regions of rural India. In another work, Eaton et al. (1981) applied the maximal covering model to located ambulance bases and centres from which to recruit rural health workers in Colombia. Another study conducted in India used covering tour models to determine planning mobile health services in the Salcette region of Goa (Hodgson et al., 1998). In Rio, Brazil, Galvão et al. (2002) strategically determined the location of healthcare facilities in order to optimize maternal and perinatal services. Bapna et al. (2002) also applied location modelling in India to determine facilities settings of unleaded petrol stations using optimization techniques. In a study in Brazil, public schools' locations were determined for urban areas using locational analysis and GIS (Pizzolato et al., 2004). Yasenovskiy and Hodgson (2007) presented applications pertaining to the African country Ghana, for determining the location of healthcare provision bases in rural areas. They proposed a model that incorporates a spatial choice interaction model attributing attendance and benefits to facility size, distance, and neighbourhood accessibility. Massam and Malczewski (2016) worked on determining the location of health centres in a rural region of Zambia using a decision support system. Another study presented a more general case for planning of community health schemes by non-governmental or faith-based organizations in rural areas of developing countries, from both top-down and ground level viewpoints (Smith et al., 2009). Rahman and Smith (2000) provide a review of location-allocation models in planning for healthcare facilities, presenting different applications of mathematical models. They highlight the differences between developed countries and emerging 
markets stating that poor geographical accessibility is the main reason why basic health care does not reach the majority of the population in emerging markets. Examples of their applications pertained to locating hospitals and ambulance stations. In their review, Rahman and Smith (2000) state that although existing mathematical models of facility location are too sophisticated for use in emerging markets because such setting can present barriers in implementation. These models have demonstrated usefulness in the facility location decision-making process; however, they can face political and economic barriers. The literature review shows that facility modelling has been implemented in different ways in emerging markets, taking specific characteristics that are relevant to each case. The main differences in facility location between emerging markets and developed countries are related to geographical configuration (difference between urban and rural areas), geographical accessibility, the sector of activity, and nature of facilities. Although these differences have been highlighted in the literature, their impact on the location of facilities has not been studied. Table 1 presents a summarized comparison between emerging markets and developed countries based on characteristics that can affect facility location decisions. The characteristics under comparison are: geographical configuration, physical infrastructure, technological infrastructure, availability of information, funds and resources utilization, and market maturity.

Several studies have suggested the negative effects of emerging market characteristics on supply chain management related to the lower quality and availability of infrastructure and resources play an important role in the selection of strategic locations (Lorentz et al., 2013). Emerging markets also present differences compared to developed countries concerning geographical accessibility caused by the differences between rural and urban areas' infrastructures (Khanna and Palepu, 2013). These markets are generally more rural and present a big gap between rural and urban areas regarding accessibility and resources whereas developed countries are highly urbanized (Lorentz et al., 2013). This gap between rural and urban areas also affects demand dispersion of facilities as urban areas are more populated. Facility location models present in the literature take into consideration different influencing factors such as opening costs, transportation costs, inventories, demand volumes. In our literature study, we did not come across models that use the road infrastructure type explicitly as an input parameter when designing the network. In addition, the literature on facility location models in emerging markets shows that models have been mainly developed based on problems that exist in developed countries which is not particularly useful when dealing with the case of an emerging market failing to take into consideration the differences ins road infrastructure types and demand dispersion. While applications of facility location models to emerging markets have taken into consideration characteristics of these countries, no attention has been given to demonstrating how these differences affect facility location results. This study aims at determining how differences such as road infrastructure type and demand dispersion affect the results of facility location with an application to a case study of the Moroccan public pharmaceutical supply chain where these characteristics may present an issue.

\section{MODEL DESCRIPTION}

Our solution approach consists of determining the candidate locations for facilities first and determining optimal locations by solving the developed mathematical model. The first step of the proposed model approach is to determine candidate locations for facilities through geographical modelling. For the purposes of this study, we selected the set covering model and adapt it to take into consideration characteristics of emerging markets. The investigation of literature concerning the specificities of emerging markets led to choosing road infrastructure type and demand dispersion as the main characteristics on which the coverage distance would depend. The aim is to assess how these characteristics affect the location modelling in the case of an emerging market. Solving the problem allows determining the optimal number and location of facility locations resulting in a network configuration that satisfies the different requirements of the problem. Finally, we conduct a sensitivity analysis where different scenarios are built to show how the number of warehouses and the network configuration change when varying coverage distances taking into consideration road infrastructure type and demand dispersion.

\subsection{Determining the Candidate Locations}

The first step of the solution approach is to discretize the location problem to determine candidate locations. Typically, location problems in continuous spaces are modelled using nonlinear optimization problems (where the nonlinearity derives from the Euclidean-distance function), it is shown how standard covering problems can be reduced so as to allow a discrete search (Eiselt, 2004). Therefore, instead of iteratively searching for good solutions in continuous location problems, like many algorithms or heuristics do, it is suggested to identify a finite set which consists of at least one optimal solution. This finite set is known as the Finite Dominating Set (FDS) (Murray et al., 2008). By examining the finite set, instead of the infinite space, search effort can be reduced dramatically. Hakimi (1964, 1965) was the first to investigate the FDS for a network location problem. He proved that nodes of the network comprise an FDS for the p-median problem.

Church (1984) developed an approach for identifying the FDS for covering problems involving point-based demand for both Euclidean distance and rectilinear distance. The FDS is a set of intersection points of the circles with radius of the service standard centred on the demand locations, known as the circle intersection point set (Figure 1). In order to find the FDS for this model, we use GIS (Geographic Information System) modelling tools to create buffers around demand centres with distances measured on the road network. The FDS can be reduced using aggregation methods. The output of this process is a set of coordinates for candidate locations. 
Table 1 Differences Between Emerging Markets and Developed Countries

\begin{tabular}{|c|c|c|}
\hline Characteristics & Main differences & References \\
\hline Geographical configuration & $\begin{array}{l}\text { Emerging markets are generally more rural and present a big } \\
\text { gap between rural and urban areas whereas developed } \\
\text { countries are highly urbanized }\end{array}$ & Lorentz et al. (2013) \\
\hline Physical infrastructure & $\begin{array}{l}\text { Developed Countries have good infrastructure including roads } \\
\text { and transportation means. Whereas the quality and availability of } \\
\text { this infrastructure is moderate to poor in emerging markets. }\end{array}$ & $\begin{array}{l}\text { Ibrahim and Damasceno (2012) } \\
\text { Smith et al. (2009) } \\
\text { Yasenovskiy and Hodgson (2007) }\end{array}$ \\
\hline Technological infrastructure & $\begin{array}{l}\text { Developed countries have higher technological capabilities, } \\
\text { available telecommunications networks and communication flow } \\
\text { systems compared to emerging countries. }\end{array}$ & $\begin{array}{l}\text { Arney et al. (2014) } \\
\text { Hlyal, et al. (2015) }\end{array}$ \\
\hline Availability of information & $\begin{array}{l}\text { Emerging markets have less available information and struggle } \\
\text { with data limitation compared to developed countries. }\end{array}$ & Drezner and Hamacher (2001) \\
\hline $\begin{array}{l}\text { Funds and resources } \\
\text { utilization }\end{array}$ & $\begin{array}{l}\text { Resources are effectively and efficiently utilized in developed } \\
\text { countries whereas this utilization of resources is poor in } \\
\text { emerging markets. }\end{array}$ & (Rahman, 2000) \\
\hline Market maturity & $\begin{array}{l}\text { Developed Countries are prosperous compared to emerging } \\
\text { markets which are on the path of industrialization. }\end{array}$ & $\begin{array}{l}\text { Khanna and Palepu (2013) } \\
\text { Kvint (2009) }\end{array}$ \\
\hline
\end{tabular}

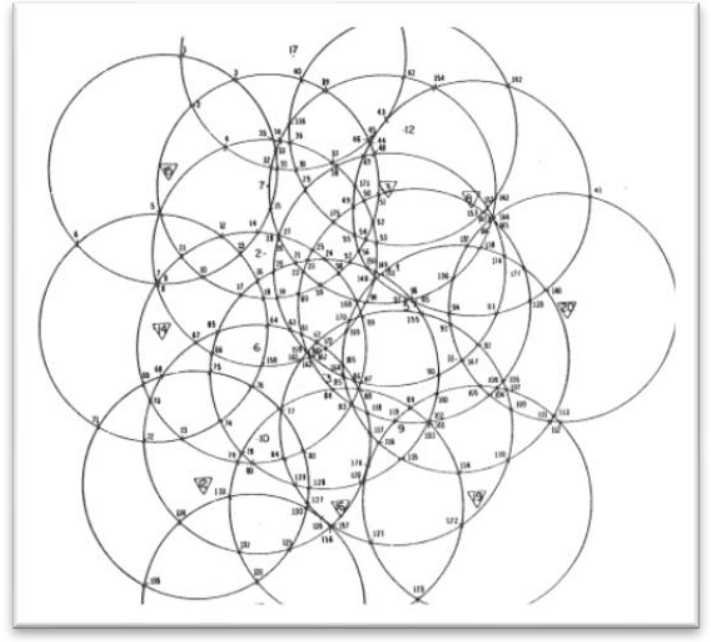

Figure 1 Circle Intersection Point Set (Mehrez, 2016)

\subsection{Determining Optimal Locations}

The second step of our approach is the mathematical modelling of the problem with the objective to select the optimal facility locations from the candidate locations. Following the mainstream in the literature, we opted for a set covering model (Farahani et al., 2012). The problem seeks to identify a minimal set of facilities and their locations so that all demand points are covered. The objective is to determine the location of facilities to cover a given set of demand points, such that each demand point is served by a facility within a maximum service distance $R_{j}$. The set covering model minimizes the opening cost of the facilities that are selected such that all demand nodes are covered. The special feature of this model is that the coverage distance $R_{j}$ is a function that takes into consideration the emerging market characteristics. The coverage distance $\mathrm{R}_{\mathrm{j}}$ will vary according to the demand points' dispersion and road infrastructure type. Demand priority is represented by an index $P_{j}$ that can take values 1 to $\mathrm{m}$. For example, demand can be categorized to high, medium, or low priority thus resulting in 3 varying values for $R_{j}$. In the same manner, the road infrastructure type $T_{j}$ is an index that can take value 1 to n. For example, these categories can be good, medium, and bad road infrastructure type. When taking into consideration both demand dispersion and infrastructure type, $R_{j}$ becomes a matrix of $n * m$ values. The mathematical formulation of the model is presented below.

\section{Input parameters}

$J$ : Set of demand points

$I:$ Set of candidate locations

$D_{i j}$ : Distance from facility $i$ to demand point $j$

$f_{i}$ : Opening cost of facility $i$

$P_{j}$ : Category index for demand priority of demand point $j$ $P j=[1, \ldots m], \quad m=$ number of categories

$T_{j}=$ Category index for road infrastructure type of demand point $j$

$T j=[1, \ldots n], \quad n=$ number of categories

$d\left(P_{j}, T_{j}\right)=$ Coverage distance function for index $P_{j}$ and index $T_{j}$

$d\left(P_{j}, T_{j}\right)=\left[\begin{array}{cccc}d_{11} & d_{12} & \ldots & d_{1 m} \\ d_{21} & d_{22} & \ldots & d_{2 m} \\ \ldots & \ldots & \ldots & \ldots \\ d_{n 1} & d_{n 2} & \ldots & d_{n m}\end{array}\right]$

$R_{j}=d\left(P_{j}, T_{j}\right)=$ Coverage distance required by demand

$a_{i j}=\left\{\begin{array}{c}1 \text { if } \text { Dij } \leq \text { Rj } \\ 0 \text { otherwise }\end{array}\right.$ point $j$

\section{Decision variable}

$X_{i}=\left\{\begin{array}{c}1 \text { if we locate the warehouse at candidate location } i \\ 0 \text { otherwise }\end{array}\right.$

\section{Optimization model}

Minimize $\quad \sum_{i \in I} f_{i} X_{i}$
Subject to:
$\sum_{i \in I} a_{i j} X_{i} \geq 1$
$\forall j \in J$

The objective function (1) minimizes the total facilities' cost. Constraint (2) stipulates that each demand node must be covered by at least one of the selected facilities. Finally, constraint (3) ensures that $X_{i}$ is a binary variable. Kindly note that $\mathrm{Rj}$ is a function of the parameters $\mathrm{Pj}$ and $\mathrm{Tj}$, which denote the 'demand priority' and 'road infrastructure type' respectively, which affect the optimal facility locations via the parameters $a_{\mathrm{ij}}$. 


\section{CASE STUDY}

The model presented earlier has been applied to the case study concerning the redesign of the Moroccan public pharmaceutical supply chain. Pharmaceutical products represent nearly $40 \%$ of the overall government's expense of healthcare in Morocco. In the public sector, the Ministry of Health has allocated in 2013, more than two billion USD in medicines and health care products. However, despite efforts to date in connection with the supply of drugs and medical devices in terms of cost reduction (joint purchasing) and quality assurance (norms and standards), the availability of these products in public health institutions is worrying as patients do not fully benefit from these efforts ( $\mathrm{MOH}$ report, 2014). Since the expansion of the medical assistance scheme in 2012, a large portion of the Moroccan population has access to free medical care and services; which resulted in a significant increase of the number of patients and purchased pharmaceuticals. At present, the annual budget allocated for the purchase of pharmaceuticals is over 200 million USD; this amount has increased by nearly $567 \%$ over a 10 years period. Several assessments of the supply chain of the current pharmaceutical products demonstrated the saturation of the logistical system and its inadequacy to the new challenges faced. Given that the distribution system has not experienced significant growth for the past decades, it has now become saturated due to a lack of capability to effectively manage and distribute the current amount of pharmaceuticals. Several operational problems associated with the distribution network have erupted. They include stock-outs that takes a long time to replenish, the expiry rate of pharmaceuticals not delivered or used, the unavailability of these products at the right time and the right place patients, and the lack of responsiveness of the network design. These problems are mainly caused by the fact that there is a central distribution configuration which delays the distribution process towards the numerous demand points. One significant inconsistency in the supply chain is then related to the centralized storage system. The aim of this study is to suggest a redesign of the pharmaceutical storage system by applying our model to increase the reactivity of the supply chain.

Figure 2 presents the structure of the Moroccan public pharmaceutical supply chain. The system includes a number of product suppliers who deliver to one main warehouses (holding $80 \%$ of the total inventory) and 3 secondary ones. These warehouses are all located in the region of Casablanca, Morocco's biggest city and the economic capital of the country. Pharmaceutical products are then delivered to public pharmacies that are located in 78 provincial and regional hospitals, and 83 provincial health delegations which deliver to 2759 basic health centres (ESSP) geographically distributed over the country. All products are distributed by small trucks or small vans, especially upstream in the supply chain. At the downstream level, that is the distribution to the provincial hospitals, delegations, and basic healthcare facility, smaller vans and cars are typically used as transportation mode. The majority of delegations happen to be located right next to regional or provincial hospitals. Information flow pertaining to orders and distribution management happens between the supply division and demand points (hospitals and delegations), as well as pharmaceutical suppliers.
As an emerging market, Morocco's geographical structure shows differences between urban and rural areas. Facility location applications to the public sector and healthcare in Morocco have not been addressed in the literature before. In fact, the models pertaining to the Moroccan context are not useful to our case study as they have been concerned with small scale areas of either the private sector or the specific case of renewable energies, and they do not demonstrate the impact of emerging market characteristics on facility location results.

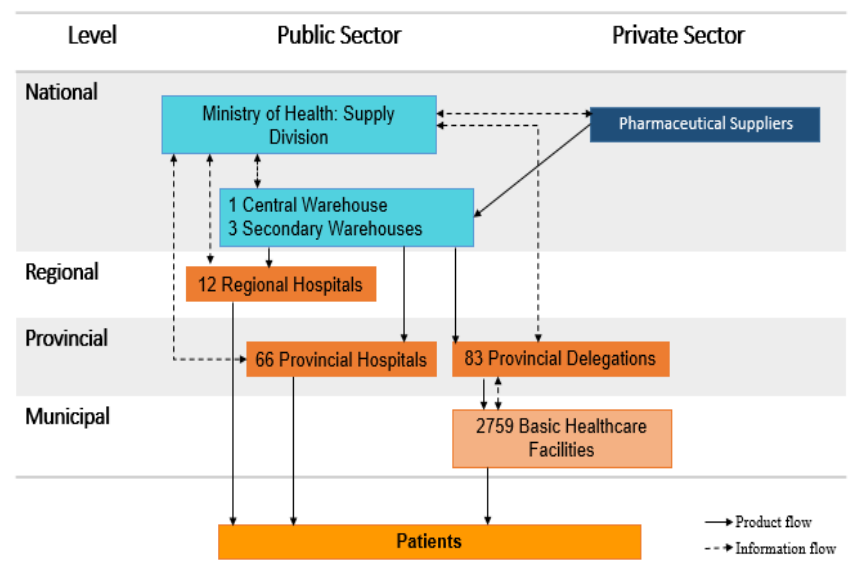

Figure 2 Pharmaceutical Supply Chain Structure

\subsection{Data Collection}

Data for this study were provided by the Ministry of Health in Morocco. We started with a study and analysis of the existing distribution network on the physical and information systems, and then collected data related to demand quantities, which were shared by the Ministry of Health. We were able to conduct multiple interviews with the Ministry of Health personnel in different sites such as warehouses and hospital pharmacies. It is the Procurement Division of the Ministry of Health that is responsible for supplying pharmaceuticals to the public sector. Its mission is to ensure the execution of expenditure of pharmaceutical products to Directions, Delegations, Hospitals and Healthcare Centers. These pharmaceutical products occupy a considerable place in the Moroccan health system, they alone account for $31.7 \%$ of total health expenditure (National Health Accounts, 2013). The demand data that we obtained include a database of all medicines that the government supplies to patients via the regional and provincial hospitals and delegations. The supply of pharmaceutical products expressed by the 158 health units in the country (including 81 delegations with 2,750 basic healthcare establishments and 144 hospitals) is met by the delivery of around 25000 tons, comprising a number of units between 40 and 50 million boxes coming from more than 60 suppliers and service providers. Pharmaceuticals contain over 1050 references of medicines, medical devices and laboratory reagents. These medicines include pain relief, vaccinations, antibiotics, and other basic medicines which the government considers important to be available to all citizens. The ministry also shared the number and exact locations of all healthcare centers. On the other hand, data concerning road infrastructure was retrieved from the Moroccan Ministry of Equipment and Transportation. 
The calculation of the opening cost $f_{i}$ is based on the land price and construction costs per squared meter for a typical pharmaceutical warehouse. Existing facilities were given an opening cost of $f_{i=0}$. For other candidate locations, land price depends on their locations and was retrieved from the real estate price benchmarks provided by the General Tax Directorate of the Ministry of Economy and Finance. Construction costs, on the other hand, are considered equal across the country.

The priority index $P j$ of demand points was determined based on the demand quantity for pharmaceuticals for each healthcare centre. Demand points that serve a large portion of the population require larger quantities of pharmaceutical products and have a high turnover rate. Consequently, replenishment of these demand points requires a higher number of trips to deliver the products, which results in higher transportation costs. Demand data for hospitals and delegations is available as an aggregate sum of the number of boxes. For the purposes of this case study, the priority index has been set equal to 3 different values: "high priority", "medium priority", and "low priority". The cut-off points were selected dependent on the ranking of the demand volume of each demand point, ensuring a more or less equal number of demand points in each category. In order to determine the priority index of a demand point, the following steps have been performed:

1- Aggregate demand values for regional/provincial hospitals and delegations. This resulted in a total of 81 demand points.

2- Rank these demand values in descending order with (1) being highest demand and (81) the lowest.

3- Determine the cut-off points for the priority index $P j$, by subdividing the ranking to 3 parts of equal sides:

$$
P_{j}=
$$

(1 "High priority" if demand ranking is from (1) to (27)

2 "Medium priority" if demand ranking is from (28) to (54)

3 "Low priority" if demand ranking is from (55) to (81)

We are aware that different cut-off points could have been selected but we believe that by doing so, at least, we ensure that some priority is given to demand point areas where demand and consumption of pharmaceuticals is high and requires frequent replenishment.

The road Infrastructure type of demand points was determined from the data gathered concerning road networks in Morocco from the Ministry of Equipment and Transportation. This data compares the Moroccan regions with values for the number of $\mathrm{km}$ of paved roads, non-paved roads, and their percentages compared to total national roads for every location. The road infrastructure RI ratio has been calculated as follow:

RI Ratio (for each region)

$$
=\frac{\text { Number of road } \mathrm{kms} \text { nonpaved }}{\text { Number of road kms paved }}
$$

When calculating the RI ratio for all regions in the country, it enabled us to determine two cut-off points (RI = $15 \%$ and $\mathrm{RI}=40 \%$ ) for the RI ratio. Therefore, a region with an RI ratio less than $15 \%$ was considered to have a good road type and a RI ratio that exceeds $40 \%$ represented a region with bad road type, values in between were classified as a region medium quality of road infrastructure. Finally, the road type index $\mathrm{Tj}$ takes three values according to the RI ratio distribution as shown hereafter:

$$
T_{j}=\left\{\begin{array}{lll}
1 & \text { if } \quad \boldsymbol{R I} \leq \mathbf{1 5} \% \\
2 & \text { if } \quad \mathbf{1 5} \%<\boldsymbol{R I} \leq \mathbf{4 0} \% \\
3 & \text { if } \quad \boldsymbol{R I}>\mathbf{4 0} \%
\end{array}\right.
$$

\subsection{Results}

The purpose of the model is to determine the optimal locations of warehouses to cover all healthcare demand points such that each one is served by a facility within a maximum coverage distance. The mathematical model is modelled in the General Algebric Modeling Systems (GAMS) software and solved using Cplex solver. The use of GIS modelling tools in conjunction with the optimization program was used for computing data to determine distances and create buffers, or areas of influence, around centres with distances measured on the road network.

\subsubsection{Determining candidate locations}

The GIS system was used to find candidate locations according to the coverage distance using intersection points of the buffers in addition to the current location of hospitals. In order to reduce the number of candidate locations, intersection points that fall into a small perimeter were aggregated. Figure 3 shows the buffers (3a), the intersection points and the areas of aggregation (3b) and the final candidate locations $(3 \mathrm{c})$, respectively.

\subsubsection{Determining optimal warehouse locations}

The set covering model presented in section 3.2 is applied to the Moroccan facility location case with sets of 81 demand points and 49 candidate locations. An integer optimal solution was found by using the branch and cut algorithm. The distances between candidate locations and demand points were calculated according to the Vincenty formula. There are several ways to calculate great circle distances, including spherical law of cosines, Harversine (Earth as sphere), and Vincenty (Earth as Ellipsoid) formulas. When dealing with populated areas, the rectilinear (Manhattan distance) is more accurate than the Euclidean distance. However, when calculating distances over large areas or using latitude and longitude coordinates, distance measurements should be calculated along the curved surface of the earth (great circle distance) (Boscoe, 2013). The Vincenty formula is the most accurate up to date formula that calculates distances between a pair of latitude/longitude points on the earth's surface, using an accurate ellipsoidal model of the earth. The distance between candidate location and demand point for our model was calculated using the Vincenty formula (See Appendix A.)

In order to evaluate the model, we created 3 sets of numerical experiments: a base scenario and 2 other sets where demand dispersion and infrastructure type are taken into consideration respectively. The base scenario represents 

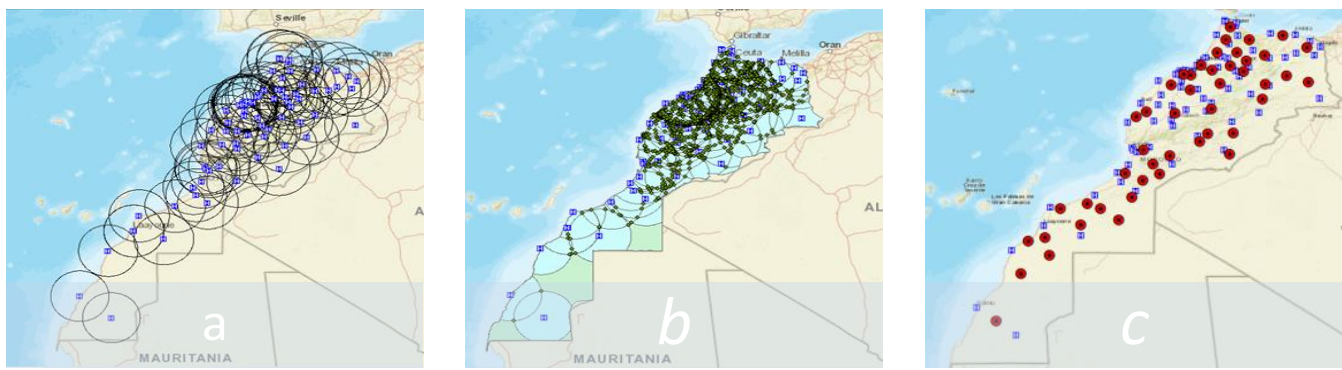

Figure 3 Candidate Warehouses Location

the classic set covering with an identical coverage distance for all demand points. Road type and demand dispersion are not taken into account in this case. By this first set of results (Table 2), we want to portrait scenarios where $150 \mathrm{~km}$ is the average coverage distance. The optimal configuration of the base scenario is illustrated in Figure 4. We can see that 11 warehouses will be opened and distributed evenly around the areas where hospital centres are located.

Table 2 Summary of Results

\begin{tabular}{lc}
\hline \multicolumn{1}{c}{ Scenario } & Number of warehouses \\
\hline Scenario 1 : Base scenario & 11 \\
\hline Scenario 2 & 15 \\
\hline Scenario 3 & 16 \\
\hline
\end{tabular}

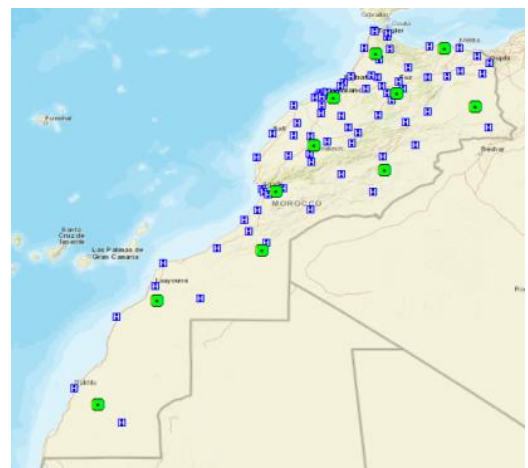

Figure 4 Optimal Warehouse Location for Base Scenario

In the second scenario, demand dispersion was taken into consideration and the coverage distance is set to $150 \mathrm{~km}$ with a range of $100 \mathrm{~km}$. That means that the distances for high, medium, and low demand dispersion are 100, 150, and $200 \mathrm{~km}$ respectively. Figure 5 shows the results where 15 warehouses are built taking into consideration different demand priorities. Compared to the base scenario, more warehouses are suggested in the south of Morocco.

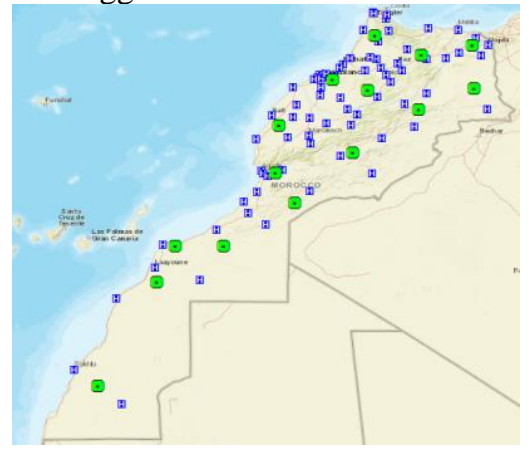

Figure 5 Optimal Warehouse Location for 2nd Scenario

In the third scenario where infrastructure type is taken into consideration, 16 warehouses opened. Figure 6 shows the locations of these warehouses. We can see that the warehouses are closer to areas where infrastructure is bad, and further from the areas where road infrastructure is good such as the far north or the northern east of Morocco. On the other hand, the southern part has a lower infrastructure quality compared to the north, which explains the location of warehouses closer to demand points.

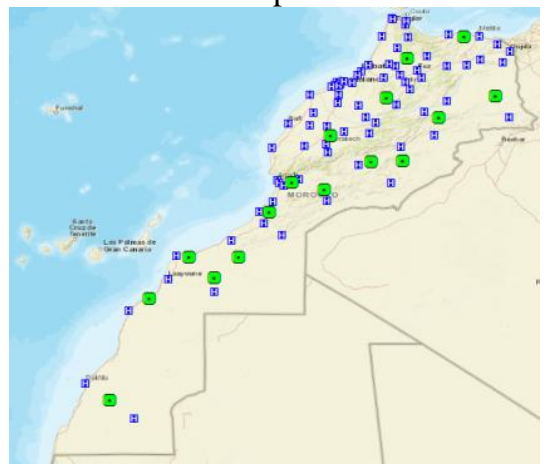

Figure 6 Optimal Warehouse Location for $3^{\text {rd }}$ Scenario

\subsubsection{Sensitivity analysis}

In this section, we present the sensitivity analysis. We are interested in knowing how coverage distance, demand dispersion, and infrastructure type impacts the optimal network configuration. Scenarios were first constructed around the base scenario varying the coverage distance from $150 \mathrm{~km}$ to $400 \mathrm{~km}$. Taking into consideration demand prioritization and road infrastructure type, 8 scenarios were constructed where coverage distance varies from $150 \mathrm{~km}$ to $350 \mathrm{~km}$ while the range difference is either 100 or $200 \mathrm{~km}$. Table 3 summarizes the experimental design and presents the optimal number of warehouses. By varying the coverage distance, not taking into consideration demand and infrastructure type. Figure 7. shows how the number of warehouses varies as the coverage distance decreases (Figure 7).

\section{Number of warehouses vs. Coverage distance}

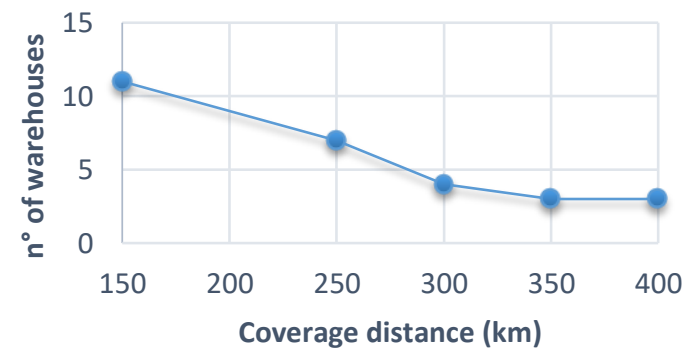

Figure 7 The Relationship Between the Number of Warehouses and the Coverage Distance 
Figure 8 illustrates the results of scenarios 8, 9, 12, and 13, which take into consideration demand dispersion with a coverage distance varying from $150 \mathrm{~km}$ to $350 \mathrm{~km}$ and a range of $100 \mathrm{~km}$. The results show that warehouses shift to urban areas when demand is high. In addition, the number of warehouses varies as the coverage distance is varied as shown in Figure 9.

Another set of 8 scenarios were constructed by varying the road infrastructure type along with the coverage distance from 150 to $350 \mathrm{~km}$ and the range, taking values of 100 and
$200 \mathrm{~km}$ (Figure 9 and 10). The results show how the optima number of warehouses changes when the coverage distance is varied. The number of warehouses becomes larger when the range is high. Except when the number of warehouses reaches 3 , then the range doesn't show any difference in the results. The map representation in Figure $\mathbf{1 1}$ shows the results for scenarios $16,17,20$, and 21 where the range is 100. The location of warehouses shifts to regions that are less urbanized since they present accessibility priority due to a smaller coverage distance.

Table 3 Summary of the Experimental Design and Result

\begin{tabular}{|c|c|c|c|c|c|}
\hline Scenario & $\begin{array}{c}\text { Average } \\
\text { coverage } \\
\text { distance }(\mathrm{km})\end{array}$ & $\begin{array}{l}\text { Distance } \\
\text { Range (km) }\end{array}$ & $\begin{array}{c}\text { Demand } \\
\text { dispersion } \\
(Y e s=1, \mathrm{No}=0)\end{array}$ & $\begin{array}{c}\text { Infrastructure Type } \\
\left(Y e s=1, N_{0}=0\right)\end{array}$ & $\begin{array}{c}\text { Number of } \\
\text { warehouses }\end{array}$ \\
\hline 1 & 150 & 0 & 0 & 0 & 11 \\
\hline 2 & 250 & 0 & 0 & 0 & 7 \\
\hline 3 & 300 & 0 & 0 & 0 & 4 \\
\hline 4 & 350 & 0 & 0 & 0 & 3 \\
\hline 5 & 400 & 0 & 0 & 0 & 3 \\
\hline 6 & 150 & 100 & 1 & 0 & 15 \\
\hline 7 & 200 & 200 & 1 & 0 & 12 \\
\hline 8 & 200 & 100 & 1 & 0 & 9 \\
\hline 9 & 250 & 100 & 1 & 0 & 7 \\
\hline 10 & 250 & 200 & 1 & 0 & 8 \\
\hline 11 & 300 & 200 & 1 & 0 & 7 \\
\hline 12 & 300 & 100 & 1 & 0 & 5 \\
\hline 13 & 350 & 100 & 1 & 0 & 3 \\
\hline 14 & 150 & 100 & 0 & 1 & 16 \\
\hline 15 & 200 & 200 & 0 & 1 & 14 \\
\hline 16 & 200 & 100 & 0 & 1 & 8 \\
\hline 17 & 250 & 100 & 0 & 1 & 6 \\
\hline 18 & 250 & 200 & 0 & 1 & 7 \\
\hline 19 & 300 & 200 & 0 & 1 & 5 \\
\hline 20 & 300 & 100 & 0 & 1 & 5 \\
\hline 21 & 350 & 100 & 0 & 1 & 4 \\
\hline 22 & 300 & 100 & 1 & 1 & 5 \\
\hline 23 & 350 & 200 & 1 & 1 & 5 \\
\hline 24 & 450 & 200 & 1 & 1 & 4 \\
\hline 25 & 450 & 400 & 1 & 1 & 6 \\
\hline
\end{tabular}



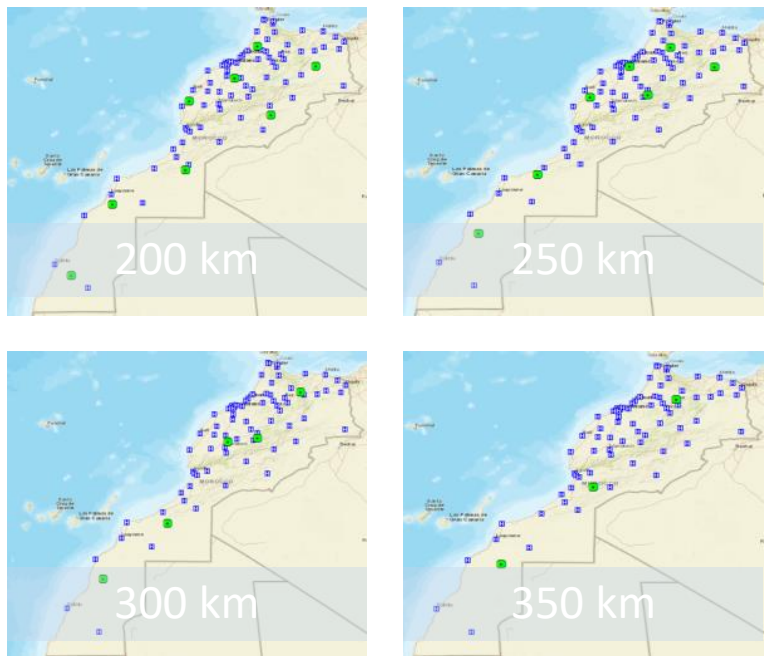

Figure 8 Map Representation of Warehouses Based on Demand Dispersion (Scenarios 8, 9, 12, and 13)

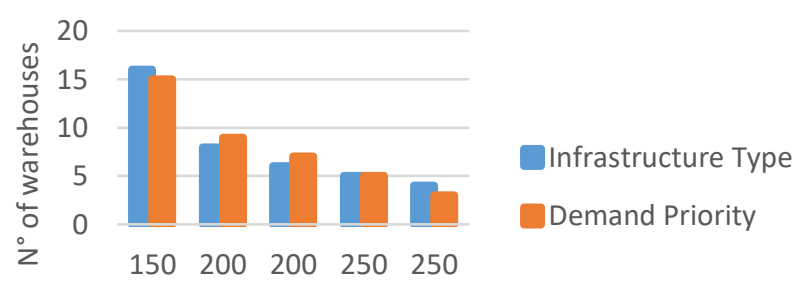

Coverage distance $(\mathrm{km})$

Figure 9 Number of Warehouses for Range $100 \mathrm{Km}$

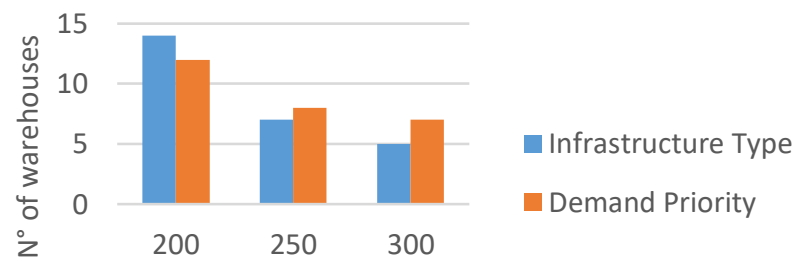

Coverage distance $(\mathrm{km})$

Figure 10 Number of Warehouses for Range $200 \mathrm{Km}$
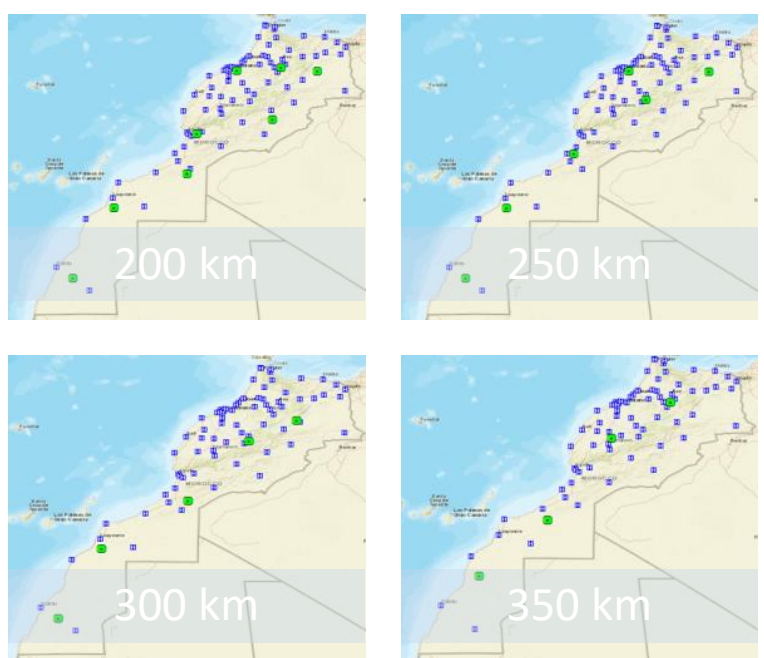

Figure 11 Map Representation of Warehouses Based on Infrastructure Type (Scenarios 16, 17, 20, and 21)
We also investigated scenarios 22 to 25 where both demand dispersion and infrastructure type are combined in the coverage distance function. This distance then depends on both demand dispersion and road infrastructure type. The proposed example shows a matrix having "high, medium, low priority" for demand and "good, medium, bad" for road types. Tables 4 to 7 present coverage distance values used to select the appropriate coverage distance depending on the two factors. The results of these scenarios are presented in Figure 12. The results for scenario 22 , where the coverage distance varies between 200 and $400 \mathrm{~km}$, give 5 optimal locations, 4 of them located in the northern part of the country. This can be explained by the high demand of public health centers in that region compared to the south, which is geographically less populated. In scenario 23 , the coverage distance varies between $150 \mathrm{~km}$ and $550 \mathrm{~km}$ with a $350 \mathrm{~km}$ average and a $200 \mathrm{~km}$ range. We notice that the number of warehouses increases in the south part of the country compared to the northern west part where demand is high because road infrastructure type is good in these areas where most big cities are located. In scenario 24 , the coverage distance increases and the number of optimal warehouses decreases to 4 . When we increase the range and the coverage values vary between $150 \mathrm{~km}$ and $850 \mathrm{~km}$, the results show that the number of warehouses increases. This can be explained by the fact that the number of areas where the road infrastructure type is bad and medium outweighs areas that have good road infrastructure.

Table 4 Coverage Distance Values for Scenario 22

\begin{tabular}{cccc}
\hline Road Infr. & $\begin{array}{c}\text { Demand Priority } \\
\text { (HIGH) }\end{array}$ & $\begin{array}{c}\text { Pj=2 } \\
\text { (MEDIUM) }\end{array}$ & $\begin{array}{c}\text { Pj=3 } \\
\text { (LOW) }\end{array}$ \\
\hline $\mathrm{Tj}=1(\mathrm{GOOD})$ & $300 \mathrm{~km}$ & $350 \mathrm{~km}$ & $400 \mathrm{~km}$ \\
\hline $\mathrm{Tj}=2(\mathrm{MEDIUM})$ & $250 \mathrm{~km}$ & $300 \mathrm{~km}$ & $350 \mathrm{~km}$ \\
\hline $\mathrm{Tj}=3(\mathrm{BAD})$ & $200 \mathrm{~km}$ & $250 \mathrm{~km}$ & $300 \mathrm{~km}$ \\
\hline
\end{tabular}

Table 5 Coverage Distance Values for Scenario 23

\begin{tabular}{cccc}
\hline \multicolumn{1}{c}{ Demand Priority } & $\begin{array}{c}\text { Pj=1 } \\
\text { (HIGH) }\end{array}$ & $\begin{array}{c}\text { Pj=2 } \\
\text { (MEDIUM) }\end{array}$ & $\begin{array}{c}\text { Pj=3 } \\
\text { (LOW) }\end{array}$ \\
\hline $\mathrm{Tj}=1$ (GOOD) & $350 \mathrm{~km}$ & $450 \mathrm{~km}$ & $550 \mathrm{~km}$ \\
\hline $\mathrm{Tj}=2$ (MEDIUM) & $250 \mathrm{~km}$ & $350 \mathrm{~km}$ & $450 \mathrm{~km}$ \\
\hline $\mathrm{Tj}=3$ (BAD) & $150 \mathrm{~km}$ & $250 \mathrm{~km}$ & $350 \mathrm{~km}$ \\
\hline
\end{tabular}

\begin{tabular}{cccc}
\hline \multicolumn{3}{c}{ Table 6 Coverage distance values for Scenario 24 } & \\
\hline Road Infr. & $\begin{array}{c}\text { Demand Priority } \\
\text { (HIGH) }\end{array}$ & $\begin{array}{c}\text { Pj=2 } \\
\text { (MEDIUM) }\end{array}$ & $\begin{array}{c}\text { Pj=3 } \\
\text { (LOW) }\end{array}$ \\
\hline $\mathrm{Tj}=1$ (GOOD) & $450 \mathrm{~km}$ & $550 \mathrm{~km}$ & $650 \mathrm{~km}$ \\
\hline $\mathrm{Tj}=2(\mathrm{MEDIUM})$ & $350 \mathrm{~km}$ & $450 \mathrm{~km}$ & $550 \mathrm{~km}$ \\
\hline $\mathrm{Tj}=3(\mathrm{BAD})$ & $250 \mathrm{~km}$ & $350 \mathrm{~km}$ & $450 \mathrm{~km}$ \\
\hline
\end{tabular}

Table 7 Coverage distance values for Scenario 25

\begin{tabular}{cccc}
\hline Demand Priority & $\begin{array}{c}\text { Pj=1 } \\
\text { (HIGH) }\end{array}$ & $\begin{array}{c}\text { Pj=2 } \\
\text { (MEDIUM) }\end{array}$ & $\begin{array}{c}\text { Pj=3 } \\
\text { (LOW) }\end{array}$ \\
\hline Rj=1 (GOOD) & $450 \mathrm{~km}$ & $650 \mathrm{~km}$ & $850 \mathrm{~km}$ \\
\hline $\mathrm{Tj}=2(\mathrm{MEDIUM})$ & $250 \mathrm{~km}$ & $450 \mathrm{~km}$ & $650 \mathrm{~km}$ \\
\hline $\mathrm{Tj}=3(\mathrm{BAD})$ & $150 \mathrm{~km}$ & $250 \mathrm{~km}$ & $450 \mathrm{~km}$ \\
\hline
\end{tabular}



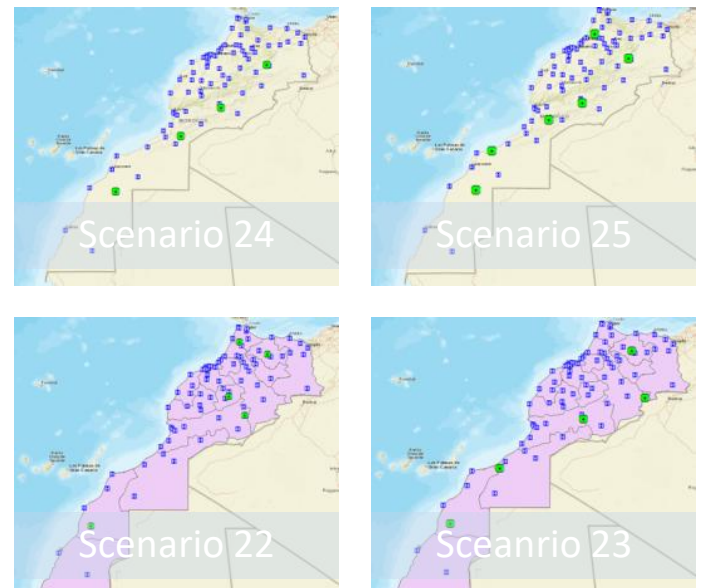

Figure 12 Optimal Warehouse Locations for Scenarios 22 to 25

The results show that the network configuration is substantially different when demand dispersion and infrastructure type are taken into consideration separately. Warehouse locations are distributed rather evenly across the country area for the base scenario. On the other hand, they tend to shift to areas where healthcare centers are closely distributed when taking into consideration demand, and further away from urban areas where infrastructure is good when taking into consideration infrastructure type. When both coverage distance and demand dispersion are varied, warehouses shift to urban areas because hospitals are mainly located in these areas. Varying the coverage distance and infrastructure type causes the warehouses to shift to regions that are less urbanized since the infrastructure type in these areas is medium to bad and therefore requires longer travel times. In the case where coverage distance is varied along with demand dispersion and infrastructure type, the number and location of warehouses depends on the average distance and the range. When the range increases, the resulting warehouses are spread all over the country, balancing urban areas in the north where demand is high and bad infrastructure type which is mainly present in the south. When the range is small, warehouses are mostly located in the northern part of the country because of the high number of public hospitals in this area, which outweighs the infrastructure bad type in the regions of the remaining hospital centres.

\section{CONCLUSION}

In this paper, we presented a facility location model that takes into consideration specific characteristics of an emerging market. The model proposes a variant of the set covering problem. The literature study concerning the specificities of emerging markets led to choosing infrastructure type and demand dispersion as characteristics on which the coverage distance would depend. The aim is to assess how these characteristics affect the location modelling. The model has been applied to a real-life case study that we conducted to redesign the Moroccan public pharmaceutical supply chain in collaboration with the Moroccan Ministry of Health. In that case study, the objective was to determine the optimal number and locations of pharmaceutical warehouses while taking into consideration different requirements of the problem. Given that the current warehouse for pharmaceuticals is saturated, the problem consists of finding the optimal locations of warehouses around the country and assigning them to national hospitals and health centres in order to ensure that the pharmaceutical product arrives to the patient at the right time and in the right quantity. The sensitivity analysis shows how the number of warehouses and the network configuration change when varying coverage distances taking into consideration road infrastructure type and demand dispersion. The results show that the number of warehouses and their location depends on the road infrastructure type and demand dispersion. Warehouses shift to urban areas where demand is high and to regions that are less urbanized since they present accessibility priority due to a smaller coverage distance. Our study shows that ignoring road infrastructure type and demand dispersion results in a substantially different solution to the network configuration. This implies that these characteristics should be taken into consideration when dealing with an emerging country setting.

Our proposed approach can help decision makers understand the importance of selecting appropriate solution approaches to solve facility location problems in emerging markets in general and in the special case of the public sector. Also, this model can be implemented by researchers and decision makers and extended and/or adapted to take into account other characteristics related to their respective problem setting. One limitation of this study was related to the unavailability of data concerning other emerging markets as the project was only applied to Morocco and more studies are needed to generalize the results. Our future research will be directed towards including other characteristics such as political and governmental constraints as well as budget constraints in the model.

\section{REFERENCES}

Amin, S. H., and Zhang, G. (2013). A multi-objective facility location model for closed-loop supply chain network under uncertain demand and return. Applied Mathematical Modelling, 37(6), pp. 4165-4176.

Arabani, A. B., and Farahani, R. Z. (2012). Facility location dynamics: An overview of classifications and applications. Computers and Industrial Engineering, 62(1), pp. 408-420.

Arney, L., Yadav, P., Miller, R., and Wilkerson, T. (2014). Strategic contracting practices to improve procurement of health commodities. Global Health: Science and Practice, 2(3), pp. 295-306.

Banerji, S., and Fisher, H. B. (1974, December). Hierarchical location analysis for integrated area planning in rural India. In Papers of the Regional Science Association (Vol. 33, No. 1, pp. 177-194). Springer-Verlag.

Bapna, R., Thakur, L. S., and Nair, S. K. (2002). Infrastructure development for conversion to environmentally friendly fuel. European Journal of Operational Research, 142(3), pp. 480496.

Batta, R., Lejeune, M., and Prasad, S. (2014). Public facility location using dispersion, population, and equity criteria. European Journal of Operational Research, 234(3), pp. 819829.

Beheshtifar, S. and Alimoahmmadi, A. (2015), A multiobjective optimization approach for location-allocation of clinics. International transactions in operational research, pp. 22: 313-328.

Boscoe, Francis P., (2013). Ed. Geographic Health Data: Fundamental Techniques for Analysis. CABI, Page 148. 
Bossert, T. J., D. M. Bowser, and J. K. Amenyah. (2003). "Is Decentralization Good for Logistics Systems? Evidence on Essential Medicine Logistics in Ghana and Guatemala." Health Policy and Planning 22: pp. 73-82.

Buor, D., 2003. Analysing the primacy of distance in the utilization of health services in the Ahafo-Ano South district, Ghana. International Journal of Health Planning and Management, 18, pp. 293-311.

Carlsson, J. G., and Jia, F. (2014). Continuous facility location with backbone network costs. Transportation Science, 49(3), pp. 433-451.

Church, R. L. (1984). The planar maximal covering location problem. Journal of Regional Science, 24(2), pp. 185-201.

Common, R., Flynn, N., and Mellon, E. (2016). Managing public services: Competition and decentralization. Elsevier.

Daskin, M. S. (2011). Network and discrete location: models, algorithms, and applications. John Wiley and Sons.

Daskin, M. S., and Dean, L. K. (2005). Location of health care facilities. In Operations research and health care (pp. 43-76). Springer US.

Drezner, Z., and Hamacher, H. W. (Eds.). (2001). Facility location: applications and theory. Springer Science and Business Media.

Eaton, D. J., Church, R. L., Bennett, V. L., Hamon, B. L., and Lopez, L. G. (1981). On deployment of health resources in rural Valle Del Cauca Colombia. Studies in the Management Sciences, 17, pp. 331-59.

Eiselt, H. A., and Marianov, V. (2011). Foundations of location analysis (Vol. 155). Springer Science and Business Media.

Fallah, H., Sadigh, A. N., and Aslanzadeh, M. (2009). Covering problem. In Facility Location (pp. 145-176). Physica-Verlag HD.

Farahani, R. Z., Asgari, N., Heidari, N., Hosseininia, M., \& Goh, M. (2012). Covering problems in facility location: A review. Computers \& Industrial Engineering, 62(1), pp. 368-407.

Francis, R. L., McGinnis, L. F., and White, J. A. (1992). Facility layout and location: an analytical approach. Pearson College Division.

Galvão, R.D., Espejo, L.G.A., Boffey, B., 2002. A hierarchical model for the location of perinatal facilities in the municipality of Rio de Janeiro. European Journal of Operational Research, 138, pp. 495-517.

Haase, K., and Müller, S. (2015). Insights into clients' choice in preventive health care facility location planning. $O R$ spectrum, 37(1), pp. 273-291.

Hakimi, S. L. (1965). Optimum distribution of switching centers in a communication network and some related graph theoretic problems. Operations Research, 13(3), pp. 462-475.

Han, X. (2014). Healthcare Facility Location and Capacity Configuration under Stochastic Demand (Doctoral dissertation, Texas A\&M University).

Hlyal, M., Ait Bassou, A., Soulhi, A., El Alami, J., and El Alami, N. (2015). Designing a distribution network using a two level capacity location allocation problem: Formulation and efficient genetic algorithm resolution with an application to a Moroccan retail company. Journal of Theoretical and Applied Information Technology, 72(2).

Hodgson, M. J., Laporte, G., and Semet, F. (1998). A Covering Tour Model for Planning Mobile Health Care Facilities in SuhumDistrict, Ghama. Journal of Regional Science, 38(4), pp. 621-638.

Ibrahim, M. M., and Damasceno, A. (2012). Hypertension in developing countries. The Lancet, 380(9841), pp. 611-619.

Khanna, T., and Palepu, K. (2013). Winning in emerging markets: A road map for strategy and execution. Harvard Business Press.

Khodaparasti, S., Maleki, H. R., Jahedi, S., Bruni, M. E., and Beraldi, P. (2016). Enhancing community based health programs in Iran: a multi-objective location-allocation model. Health care management science, pp. 1-15.
Kochetov, Y. (2011). Facility Location: Discrete Models and Local Search Methods. Combinatorial Optimization. 31, pp. $97-$ 134.

Kvint, V. (2009). The global emerging market: strategic management and economics. New York: Routledge.

Laporte, G., Nickel, S., and da Gama, F. S. (2015). Location science (p. 644). Berlin: Springer.

Lorentz, H., Kittipanya-ngam, P., and Srai, J. S. (2013). Emerging market characteristics and supply network adjustments in internationalising food supply chains. International Journal of Production Economics, 145(1), pp. 220-232.

Massam, B. H., and Malczewski, J. (2016, February). The location of health centers in a rural region using a decision support system: a Zambian case study. In Geography Research Forum (Vol. 11, pp. 1-24).

Mehrez, A. (2016). Facility Location Problems: Review, Description, and Analysis. In Geography Research Forum (Vol. 8, pp. 113-129).

Melo, M. T., Nickel, S., \& Saldanha-Da-Gama, F. (2009). Facility location and supply chain management-A review. European journal of operational research, 196(2), pp. 401-412.

Ministere de la santé au Maroc. (2014). Termes de references relatifs à l'étude de faisabilité de l'externalisation du stockage et distribution des produits pharmaceutiques aux établissements de santé du Ministère de la Santé. Rapport.

Mitropoulos, P., Mitropoulos, I., Giannikos, I., \& Sissouras, A. (2006). A biobjective model for the locational planning of hospitals and health centers. Health Care Management Science, 9(2), pp. 171-179.

Mousavi, S. M., Tavakkoli-Moghaddam, R., Heydar, M., and Ebrahimnejad, S. (2013). Multi-criteria decision making for plant location selection: an integrated Delphi-AHPPROMETHEE methodology. Arabian Journal for Science and Engineering, 38(5), pp. 1255-1268.

Mousazadeh, M., Torabi, S. A., Pishvaee, M. S. and Abolhassani, F. (2018), Health service network design: a robust possibilistic approach. International transactions in operational research, 25: pp. 337-373.

Murray, A. T., and Tong, D. (2007). Coverage optimization in continuous space facility siting. International Journal of Geographical Information Science, 21(7), pp. 757-776.

Nickel, S., and da Gama, F. S. (2015). Multi-Period Facility Location. In Location Science (pp. 289-310). Springer International Publishing.

Pizzolato, N. D., Barcelos, F. B., Lorena, N., and Antonio, L. (2004). School location methodology in urban areas of developing countries. International Transactions in Operational Research, 11(6), pp. 667-681.

Rahman, S. (1991). Location-allocation modelling for primary health care provision in Bangladesh (Doctoral dissertation, University of Exeter).

Rahman, S. U., and Smith, D. K. (2000). Use of location-allocation models in health service development planning in developing nations. European Journal of Operational Research, 123(3), pp. 437-452.

Rais, A., \& Viana, A. (2011). Operations research in healthcare: a survey. International Transactions in Operational Research, 18(1), pp. 1-31.

Revelle, C. S., Eiselt, H. A., and Daskin, M. S. (2008). A bibliography for some fundamental problem categories in discrete location science. European Journal of Operational Research, 184(3), pp. 817-848.

Roberts, M. J. (2011). Pharmaceutical reform: a guide to improving performance and equity. World Bank Publications.

Schilling, D. A., Jayaraman, V., and Barkhi, R. (1993). A review of covering problems in facility location. Location Science, 1 , 25-55. Pergamon.

Schütz, P., Stougie, L., and Tomasgard, A. (2008). Stochastic facility location with general long-run costs and convex short- 
run costs. Computers and Operations Research, 35(9), pp. 2988-3000.

Smith, H. K., Harper, P. R., Potts, C. N., and Thyle, A. (2009). Planning sustainable community health schemes in rural areas of developing countries. European Journal of Operational Research, 193(3), pp. 768-777.

Snyder, L. V. (2006). Facility location under uncertainty: a review. IIE Transactions, 38(7), pp. 547-564.

Şahin, G., and Süral, H. (2007). A review of hierarchical facility location models. Computers and Operations Research, 34(8), pp. 2310-2331.
Thunhurst, C. (2012). Public health systems analysis - the transfer of learning between developed and developing countries. Health care management science, 15(3), pp. 283-291.

Toregas, C., Swain, R., ReVelle, C., and Bergman, L. (1971). The location of emergency service facilities. Operations Research, 19(6), pp. 1363-1373.

Yasenovskiy, V., and Hodgson, J. (2007). Hierarchical locationallocation with spatial choice interaction modeling. Annals of the Association of American Geographers, 97(3), pp. 496511.

\section{APPENDIX A}

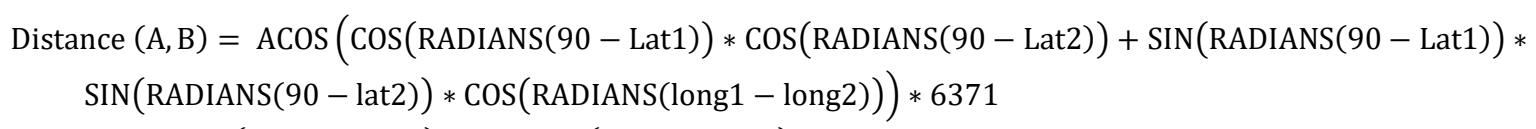

with Point $A=($ LAT1, LONG1), Point $B=(L A T 2$, LONG2) and $\mathrm{R}=$ mean radius of earth $=6371 \mathrm{~km} \quad$ (Boscoe, 2013).

Dr. Asmae El Mokrini is an assistant professor at the business school of the International University of Rabat. Her research focuses on the design of pharmaceutical supply chains destined for the public sector. She works on different projects in the field of management science with academicians and practitioners from the industry. Asmae El Mokrini earned her PhD fom the Mohammadia School of Engineers in Rabat, Morocco and the QUARTZ EA 7393 laboratory in Paris, France. She holds a Master's degree in software engineering and management science and a Bachelor's degree in engineering and management science from Al Akhawayn University in Ifrane.

Youssef Boulaksil has been a faculty member at the College of Business and Economics of the United Arab Emirates University since September 2013. Prior to that, he was a faculty member at the School of Business Administration at Al Akhawayn University in Ifrane, Morocco. He holds a MSc in Industrial Engineering and a PhD in Operations Management and Logistics from the Eindhoven University of Technology, the Netherlands. Youssef also held visiting appointments at the Nanyang Technological University (Singapore) and the Technical University of Denmark. His research focus is on supply chain management and logistics, with a focus on emerging markets. He published a number of papers in international journals, such as: International Journal of Production Economics, IISE Transactions, Interfaces, OR Spectrum, International Journal of Production Research, and International Journal of Operations and Production Management. Currently, he teaches various courses in the undergraduate program, as well as in the MBA and the MEM (Master of Engineering Management) programs. He secured in total over $\$ 400,000$ (USD) of research grants for different research projects in the last few years. For more information, see: http://faculty.uaeu.ac.ae/y_boulaksil/

\footnotetext{
Abdelaziz Berrado is Department Chair and Associate Professor of Industrial Engineering in EMI School of Engineering at Mohamed V University in Rabat. He holds a Ph.D. in Decision Systems and Industrial Engineering from ASU. His research, teaching and consulting interests are in the areas of Big Data Analytics, Industrial Statistics, Operations and Supply Chain Modelling, Planning and Control with applications in healthcare, education, manufacturing and other industries. He published several papers in research journals and conferences with local and international funding. He is a member of INFORMS, IEOM and IEEE. He was also a Senior Engineer at Intel.
} 